\title{
The Multilingual Assessment Instrument for Narratives (MAIN): Adding Cantonese to MAIN
}

\section{Angel Chan}

The Hong Kong Polytechnic University

\section{Kelly Cheng}

The Hong Kong Polytechnic University

\section{Rachel Kan}

The Hong Kong Polytechnic University

\section{Anita M.-Y. Wong}

The University of Hong Kong

\section{Roxana Fung}

The Hong Kong Polytechnic University

\section{Janice Wong}

The Hong Kong Polytechnic University

\section{Timothy Cheng}

The Hong Kong Polytechnic University

\section{Amelie Cheung}

The Hong Kong Polytechnic University

\section{Karen Yuen}

The Hong Kong Polytechnic University

\section{Barbie Chui}

The Hong Kong Polytechnic University

\section{Joyce Lo}

The Hong Kong Polytechnic University

\section{Natalia Gagarina}

Leibniz-Zentrum Allgemeine

Sprachwissenschaft (ZAS)

This paper gives an introduction to the Cantonese adaptation of Multilingual Assessment Instrument for Narratives (MAIN), which is part of the Language Impairment Testing in Multilingual Settings (LITMUS) battery. We here discuss the motivation for adapting this assessment instrument into Cantonese, the adaptation process itself and potential contexts for use of the Cantonese MAIN. 
Funded by the European Cooperation in Science and Technology, within the COST Action IS0804 "Language Impairment in a Multilingual Society: Linguistic Patterns and the Road to Assessment" (see Armon-Lotem, de Jong, \& Meir, 2015), a group of researchers from over 20 countries in Europe and beyond collaborated on a project to examine challenges that they shared in the diagnosis and assessment of multilingual children with specific language impairment (SLI; more recently Developmental Language Disorder (DLD), see Bishop, Snowling, Thompson, Greenhalgh, \& CATALISE Consortium, 2016). Specifically, the goals of this project were 1) to develop culturally-appropriate tools to assess the linguistic and cognitive abilities of bilingual children who are learning different pairs of languages, 2) to examine how bilingualism and SLI/DLD affect bilingual language development, and 3) to distinguish typically developing bilingual children from those with SLI/DLD. Major progress for the first goal was made with the development of the Language Impairment Testing in Multilingual Settings (LITMUS) battery (see Armon-Lotem, de Jong \& Meir, 2015). LITMUS includes a range of tasks examining bilingual children's development in different linguistic domains (e.g., syntax, lexicon, narrative discourse), linguistic modalities (comprehension and production), and psycholinguistic processing (e.g., nonword repetition and sentence repetition). Since the tasks took into account typological differences between languages, they were applicable for bilingual children learning a variety of languages. An example is the task for eliciting subject-verb agreement from children learning inflectional languages (de Jong, 2016). These tasks laid the foundation for achieving the second and third goals of the Action IS0804.

The Language Impairment Testing in Multilingual Settings - Multilingual Assessment Instrument for Narratives (LITMUS-MAIN, hereafter MAIN) was developed by an international team of scholars (Gagarina et al. 2012, 2015; 2019). MAIN is available in a large number of languages and is used predominantly with children aged between 3 and 12, although it has also been successfully used with teenagers up to 17 years as well as with adults (Gagarina, Bohnacker \& Lindgren, 2019). MAIN can be seen as an improvement over other existing narrative assessment tools because its design was carefully thought-out. MAIN contains four stories in two sets, with each story consisting of six pictures. Each story, as illustrated in the pictures, is grounded in the story grammar framework (Stein \& Glenn, 1979) and causal framework analysis (Trabasso \& Nickels, 1992). The two stories in each set are parallel in content and structure, allowing reliable comparison of children's narrative abilities over time, or in the two languages they speak. The stories can be elicited in two modes: story-telling and retelling. In these production tasks, the macrostructure as well as the microstructure of the children's stories can be assessed. In addition to production, comprehension of the same stories is also examined. MAIN allows researchers and speech therapy practitioners to examine, in a comprehensive fashion, the narrative competence of monolingual children as well as children who speak two or more languages, and to perform dynamic assessment. This paper describes the development and use of the Cantonese-Chinese version of MAIN. 
Until now, MAIN was primarily available in Indo-European languages. There is a need to extend the coverage of MAIN to Sino-Tibetan languages, including Cantonese-Chinese. Once available, researchers can use the Cantonese-Chinese MAIN to track the development of bilingualism in typical developing children and to identify clinical markers of SLI/DLD in bilingual children who are learning Cantonese-Chinese as one of their languages. Speechtherapy practitioners can use the Cantonese-Chinese MAIN as a criterion-referenced task to document progress in treatment for children with SLI/DLD.

This section documents our efforts in developing a Cantonese-Chinese version of MAIN. Cantonese-Chinese is a member of the Yue Chinese dialect group (of the Sino-Tibetan family) and is spoken as the lingua franca in Hong Kong, Macau, and certain places in the provinces of Guangdong (such as Guangzhou) and Guangxi in mainland China. It is also spoken by ethnic Chinese around the world including in Malaysia and Vietnam, Australia, the UK, and North America. In Hong Kong alone, more than 6 million people speak Cantonese-Chinese as their daily language (89\% of population, Census and Statistics Department, HKSAR), and worldwide there are more than 73 million speakers of Cantonese as their first language (Eberhard, Simons, \& Fennig, (eds.), 2019). Spoken Cantonese-Chinese is different from the written form (Standard Modern Chinese) that is taught in schools and used in formal contexts (Matthews \& Yip, 2011). In MAIN, the instructions for administering the narrative tasks are written in Modern Standard Chinese, but the story scripts for the story-retelling models and examples of utterances that the experimenter could use when assessing the child with Cantonese-MAIN are presented in written Cantonese as they are spoken.

Cantonese is an isolating language with the canonical word order of Subject-VerbObject (SVO). Other typological features that are particularly relevant to narratives include, for instance, topic-prominence and argument ellipsis. For a more detailed description of linguistic characteristics unique to the Chinese language, particularly those related to language difficulties that have been examined in studies on language disorders in speakers of Chinese, readers are encouraged to read Fung (2009).

MAIN was adapted into Cantonese-Chinese following the guidelines given in Bohnacker and Gagarina (2019). These guidelines clearly describe (i) the steps involved during the adaptation process; (ii) features at the macrostructural level (e.g. the number and sequence of the story components Goal, Attempt, Outcome, and Internal States for each protagonist) that must remain the same across languages; and (iii) features at the microstructural level (e.g. number of coordinating and subordinating constructions, internal state terms overall, number of direct speech sentences) that must be consistent across the stories. The adaption to Cantonese involved the concerted efforts of a team of researchers, speech-language therapists, and a research assistant led by the first author (Chan) and the final author (Gagarina), all of whom except the final author are native speakers of Cantonese residing in Hong Kong. All team members are duly recognized as co-authors. In the first phase, six student speech-language therapists studying for a Master's degree in Speech Therapy (currently all have graduated and are practicing as community speech-language therapists; sixth to eleventh authors) together 
with a research assistant (second author, Cheng)) performed the first translation of different parts of the MAIN assessment protocol into Cantonese-Chinese under the supervision of the first author (Chan) and the final author (Gagarina). The assessment protocol consists of the four-story scripts, the scoring forms, and the instructions for administration and scoring, based on the latest version of MAIN for English (Gagarina et al., 2019). The first author also interviewed about $30 \mathrm{~L} 1$ Cantonese student speech therapists, and no participants reported any cultural inappropriateness in the MAIN stories and pictures for Cantonese-speakers in Hong Kong. In the second phase, the entire or parts of the manuscript was proof-read by five expert members for accuracy and consistency. Two are developmental psycholinguists with professional qualification as speech-language therapists, currently holding a professorial position in a speech therapy training programme in two universities in Hong Kong (first author Chan and fourth author Wong). One is a Cantonese-Chinese linguist who has been working with researchers in communication disorders and holding also a professorial position at a university in Hong Kong (fifth author Fung). A postdoctoral researcher in developmental linguistics (third author Kan), and a research assistant holding a Master Degree in Linguistics (second author Cheng) are the other team members. This team effort ensures quality in the adaptation process and is needed in the dissemination of the Cantonese-Chinese MAIN to researchers who study language acquisition, Chinese linguistics or speech-language therapy and to speech-language therapists who work with bilingual children in Hong Kong and overseas.

\section{Using Cantonese-Chinese MAIN with bilingual children from South Asian backgrounds}

The Cantonese-Chinese MAIN can be used to assess competence in narrative comprehension and production in monolingual and bilingual Cantonese-Chinese children. One dominant group of non-Chinese children who acquire Cantonese-Chinese as an additional language in Hong Kong are those from South Asian ethnic backgrounds. These children usually acquire their heritage language (e.g. Urdu, Hindi, Nepali, Tagalog) as the first and family language, and Cantonese-Chinese as their second/additional, school and community language when they start attending local schools. Due to the later age at which learning of Cantonese-Chinese starts and the reduced input in this language, these bilingual children might show insufficiencies in their linguistic development which can be mistakenly diagnosed as SLI/DLD. If so, it is important to highlight the importance of assessment in both languages, to identify the direction of support in clinical decision making (see e.g. Anaya, Peña \& Bedore, 2016). Generally, there is a growing need for speech-language therapists and educators to take multilingualism and multiculturalism into account in their professional practice, as these bilingual ethnic minority children are increasingly encountered in their caseloads in recent years. Yet, there is scant research on how these bilingual Cantonese-Chinese children's speech and language develop in the Hong Kong context. Consequently, there are no tools for speech-language therapists to distinguish language difference from language impairment in bilingual Cantonese-Chinese 
children. As part of the initiative to document the language abilities of South Asian bilingual minority children in Hong Kong, and to pave the way to improve the diagnosis of SLI/DLD in these bilingual children, we assessed the narrative abilities of 24 typically-developing UrduCantonese bilinguals ( $\mathrm{M}_{\text {age }}: 9.17$ years, $\mathrm{SD}=1.68$ year) using MAIN in their two languages (Chan et al., 2018). Specifically, we asked each child to tell a story from the set of 6 pictures, then listen to the same story told according to the standard story script, and then retell that model story. Such a design allowed us to gather information about the child's responsiveness to modelling by comparing performance between the telling and the retelling conditions. The sequence of storytelling, listening to a story model, and then retelling the same story embodies the test-teach-retest paradigm of a dynamic assessment. These typically-developing bilingual children demonstrated evidence of significant improvement in the retelling condition relative to the telling condition. Such an improvement demonstrated what is called "modifiability" in dynamic assessment research. The findings are suggestive of the following: 1) evaluating a bilingual child's modifiability (improvement) upon modelling/scaffolding is important, 2) assessing modifiability is sensitive to cultural and linguistic bias against bilingual ethnic minority children, and 3) data from these typically-developing children can be used as a reference in the assessment of bilingual minority children suspected of language impairment. Future studies are needed to test the hypothesis that children with language impairment will demonstrate limited modifiability (improvement) between story-telling and retelling, relative to their typically developing peers, and children with language problems due to insufficient exposure will demonstrate comparable performance as their typically developing peers.

\section{$4 \quad$ Final remarks}

The Cantonese MAIN can be used free-of-charge for non-commercial purposes under a Creative Commons License (BY-NC-ND 3.0) provided that the copyright and licensing rules are respected. Studies that make use of this tool should cite both the assessment protocol and this introductory article as follows.

- Gagarina, N., Klop, D., Kunnari, S., Tantele, K., Välimaa, T., Bohnacker, U. \& Walters, J. (2019). MAIN: Multilingual Assessment Instrument for Narratives - Revised. Materials for use. ZAS Papers in Linguistics, 63. Cantonese version. Translated and adapted by Chan, A., Cheng, K., Kan, R., Wong, A. M-Y., Fung, R., Wong, J., Cheng, T., Cheung, A., Yuen, K., Chui, B., Lo, J. \& Gagarina, N.

- Chan, A., Cheng, K., Kan, R., Wong, A. M-Y., Fung, R., Wong, J., Cheng, T., Cheung, A., Yuen, K., Chui, B., Lo, J. \& Gagarina, N. (2020). The Multilingual Assessment Instrument for Narratives (MAIN): Adding Cantonese to MAIN. ZAS Papers in Linguistics, 64, 23-29. 
The work of Rachel Kan was supported by a Hong Kong Polytechnic University Postdoctoral Fellowship (G-YW4G) and the Hong Kong Research Grants Council Postdoctoral Fellowship Scheme (3-RA53).

\section{References}

Anaya, J. B., Peña, E. D., \& Bedore, L. M. (2016). Where Spanish and English come together: A two-dimensional bilingual approach to clinical decision making. Perspectives of the ASHA Special Interest Groups, 1(14), $3-16$.

Armon-Lotem S., de Jong J., \& Meir N. (2015). Assessing multilingual children: disentangling bilingualism from language impairment. Multilingual Matters. Bristol, UK.

Bishop, D. V. M., Snowling, M. J., Thompson, P. A., Greenhalgh, T., \& CATALISE Consortium (2016). CATALISE: A multinational and multidisciplinary delphi consensus study. Identifying language impairments in children. PLoS ONE, 11(7), e0158753.

Bohnacker, U., \& Gagarina, N. (2019). Background on MAIN - Revised, how to use it and adapt it to other languages. ZAS Papers in Linguistics, 63, iv-xii.

Census and Statistics Department, HKSAR (2018). Main table A107, 2016 Population By-census.

Chan, A., Chui, B., Lo, J., Luk, P., \& Gagarina, N. (2018). Narrative abilities of bilingual Urdu-Cantonese ethnic minority children in Hong Kong. Poster presented at the Child Language Symposium, Reading, UK, June 2018.

Eberhard, D. M., Simons, G. F., \& Fennig, C. D. (eds.). 2019. Ethnologue: languages of the world. Twenty-second edition. Dallas, Texas: SIL International. Online version: http://www.ethnologue.com.

Fung, R. S.-Y. (2009). Characteristics of Chinese in relation to language disorders. In S. P. Law, B. S. Weekes, \& A. M.-Y. Wong (Eds.). Language disorders in speakers of Chinese (pp. 1-18). Bristol, UK: Multilingual Matters.

Gagarina, N., Bohnacker, U., \& Lindgren, J. (2019). Macrostructural organization of adults' oral narrative texts. Narrative texts by children and adults: insights into their organization through a prism of language system and environmental factors. ZAS Papers in Linguistics, 62, 190-208.

Gagarina, N., Klop, D., Kunnari, S., Tantele, K., Välimaa, T., Balčiūnienė, I., Bohnacker, U., \& Walters, J. (2012). MAIN: Multilingual Assessment Instrument for Narratives. ZAS Papers in Linguistics, 56.

Gagarina, N., Klop, D., Kunnari, S., Tantele, K., Välimaa, T., Balčiūnienė, I., Bohnacker, U., \& Walters, J. (2015). Assessment of narrative abilities in bilingual children. In Armon-Lotem, S., Jong, J. d. \& Meir, N. (Eds.), Assessing multilingual children: disentangling bilingualism from language impairment (pp. 243-276). Bristol, UK: Multilingual Matters.

Gagarina, N., Klop, D., Kunnari, S., Tantele, K., Välimaa, T., Bohnacker, U., \& Walters, J. (2019). MAIN: Multilingual Assessment Instrument for Narratives. Revised version. ZAS Papers in Linguistics, 63.

Matthews, S., \& Yip, V. (2001). Cantonese: A Comprehensive Grammar (2nd ed.). London: Routledge. 
Stein, N. L., \& Glenn, C. G. (1979). An analysis of story comprehension in elementary school children. In R. O. Freedle (Ed.), New directions in discourse processing (pp. 53-120). Norwood, NJ: Ablex.

Trabasso, T., \& Nickels, M. (1992). The development of goal plans of action in the narration of a picture story. Discourse Processes, 15, 249-275. 